EPJ Web of Conferences 73, 02010 (2014)

DOI: $10.1051 /$ epjconf/20147302010

(C) Owned by the authors, published by EDP Sciences, 2014

\title{
Study of transverse momentum dependent distributions from polarised Drell-Yan at COMPASS
}

\author{
Márcia Quaresma ${ }^{a}$ on behalf of the COMPASS Collaboration \\ LIP - Laboratóio de Instrumentação e Física Experimental de Partículas, \\ Av. Elias Garcia 14-1, 1000-149 Lisboa, Portugal
}

\begin{abstract}
The Parton Distributions Functions (PDFs) and the spin structure of the nucleon are studied by the COMPASS experiment at CERN. The transverse momentum dependent (TMD) PDFs of the proton will be accessed via Drell-Yan (DY) dimuon production in negative pion collisions on a $\mathrm{NH}_{3}$ transversely polarised target. From this measurement the dimuon azimuthal asymmetries will be extracted, that can be related to 4 TMDs known as transversity, pretzelosity, Sivers and Boer-Mulders. The latter 2 TMDs are time-reversal odd distributions and are expected to change their sign when compared with the ones obtained from Semi-Inclusive Deep Inelastic Scattering, also studied in COMPASS. We aim at performing the first polarised DY measurement starting in 2014.
\end{abstract}

\section{Introduction}

The Drell-Yan process, which consists in the quark-antiquark annihilation into a virtual photon giving a lepton pair in the final state, is an excellent tool to study the TMD PDFs. It was through this process that two past experiments (NA10 [1] and E615 [2]) obtained evidences for the non-collinearity between quarks and their parent hadrons, by the measurement of a $\cos 2 \phi$ modulation up to $30 \%$. The TMD PDFs are expected to be sizeable in the valence quark region, which is probed by pion induced DrellYan off a fixed target. The DY process is well calculable, the cross-section consists in a sum of terms, each of which contains a convolution of two PDFs. The DY cross-section in LO QCD considering an unpolarised beam and a transversely polarised target can be written as:

$$
\begin{aligned}
\frac{d \sigma}{d^{4} q d \Omega}= & \frac{\alpha_{e m}^{2}}{F q^{2}} \hat{\sigma}_{U}\left\{\left(1+D_{\left[\sin ^{2} \theta\right]} A_{U}^{\cos 2 \phi} \cos 2 \phi\right)+\left|\vec{S}_{T}\right|\left[A_{T}^{\sin \phi_{S}} \sin \phi_{S}\right.\right. \\
& \left.\left.\left.+D_{\left[\sin ^{2} \theta\right]} A_{T}^{\sin \left(2 \phi+\phi_{S}\right)} \sin \left(2 \phi+\phi_{S}\right)+A_{T}^{\sin \left(2 \phi-\phi_{S}\right)} \sin \left(2 \phi-\phi_{S}\right)\right)\right]\right\}
\end{aligned}
$$

where the four azimuthal asymmetries (A), contain the PDFs convolutions.

The main drawback in using DY is its very low cross-section, which requires the use of high intensity beam in order to accumulated enough statistics. Eight quark PDFs are required to describe the nucleon

\footnotetext{
ae-mail: marcia@lip.pt
}

This is an Open Access article distributed under the terms of the Creative Commons Attribution License 4.0, which permits unrestricted use, distribution, and reproduction in any medium, provided the original work is properly cited. 


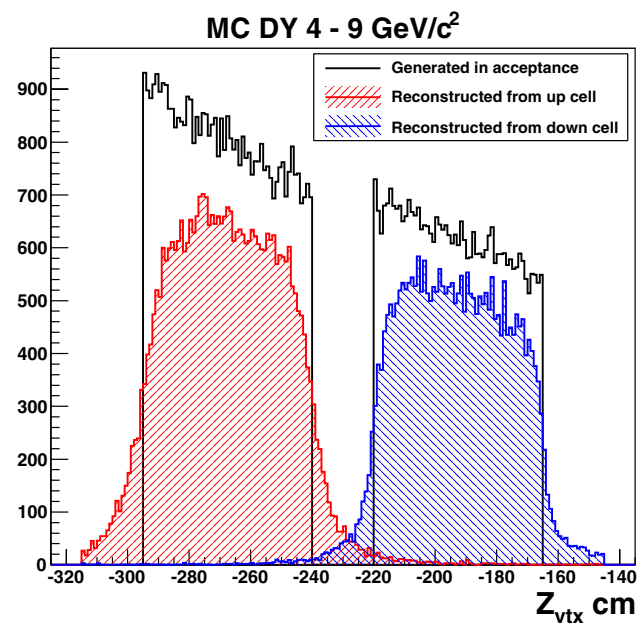

Figure 1. Distribution of primary vertex position along the beam line (only the target region is shown).

at leading twist QCD. In the COMPASS DY programme four of them will be accessed, namely BoerMulders, Sivers, transversity and pretzelosity, by the measurement of the transverse spin azimuthal asymmetries.

\section{The COMPASS Drell-Yan programme at CERN}

COmmon Muon Proton Apparatus for Structure and Spectroscopy (COMPASS) is a fixed target experiment located at the end of the M2 SPS beam line at CERN. This beam line can provide either muon or hadron beams. For the DY programme a negative pion beam with $190 \mathrm{GeV} / c$ momentum will be used. With a target made of two cells of ammonia transversely polarised with respect to the beam and in opposite directions, a polarisation of $90 \%$ can be reached. The two stages spectrometer covers a wide particles momentum range, with a geometrical acceptance of $\pm 180 \mathrm{mrad}$. The spectrometer includes a large number of tracking detectors, 2 sets of calorimeters, one RICH detector and trigger hodoscopes. A complete description can be found in [3].

The COMPASS II proposal [4] was approved by CERN for a first data taking period of 3 years including 1 year for DY, which is scheduled to start by the end of 2014 and continue in 2015. COMPASS has excellent conditions to perform the DY measurement and besides the setup is being optimised for DY. Improvements include modifications to the polarised target, a new scintillating fibres vertex detector, and modifications to the trigger hodoscopes. A hadron absorber and a beam plug will be placed upstream of the target. The main goal of this absorber is to stop the hadrons produced in the primary interactions, while the beam plug must stop the non-interacting beam. However these will also introduce multiple scattering in the dimuon signal. The absorber will be made of alumina $\left(\mathrm{Al}_{2} \mathrm{O}_{3}\right)$, which offers a good compromise between interaction lengths and radiation lengths. In addition to the absorber it is mandatory to have a proper radiation protective shielding around the target area. The primary vertices $\left(\mathrm{Z}_{\mathrm{vtx}}\right)$ resolution is quite important since asymmetries between the two target cells will be measured. A vertex detector will be placed upstream the absorber to improve the resolution. Considering this setup configuration the dimuon mass resolution is $180 \mathrm{MeV} / c^{2}$ and the $\mathrm{Z}_{\mathrm{vtx}}$ resolution is $6 \mathrm{~cm}$, which implies a very low level of contamination of events wrongly reconstructed in a given cell, as shown in Fig. 1. The feasibility of the measurement was proved by several beam tests done so far. The absorber effect 


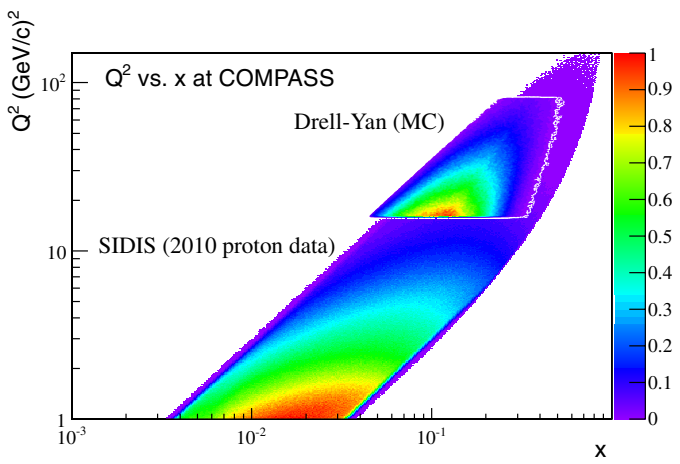

Figure 2. Phase space overlap between DY and SIDIS measurements in COMPASS.

and the spectrometer response were verified, the radiation doses measured, the detector occupancies as well as the trigger rates were checked and the dimuon trigger concept validated.

\section{Physics perspectives and projections}

COMPASS offers the unique opportunity to study Sivers and Boer-Mulders from both DY and SIDIS processes in the same experiment. These functions are time-reversal odd and for this reason they are predicted to change sign when accessed from DY or SIDIS [5]. The experimental confirmation of this sign change is considered a crucial test of the TMD factorization approach in QCD. There is a phase space overlap between the two process measurements, as seen in Fig. 2, important when comparing the extracted TMD PDFs.

There are several sources of background to consider in the DY measurement, the combinatorial background of muons from uncorrelated pion and kaon decays which is controlled by the absorber, the open charm background originated from DD̄ meson semileptonic decays, and the $\psi$ family resonances. Nevertheless the Drell-Yan signal is very clean for $M_{\mu \mu}>4 \mathrm{GeV} / c^{2}$. Even if the DY cross-section strongly decreases with the dimuon mass, the analysis will be performed in this so-called high mass region (HMR). There is also the possibility to study DY in the region $2<\mathrm{M}_{\mu \mu}<2.5 \mathrm{GeV} / c^{2}$, Intermediate Mass Region (IMR), as well as to use the $\mathrm{J} / \psi$ signal for TMD studies. In case the duality between DY and $\mathrm{J} / \psi$ stands (via the producion mechanism $q \bar{q} \rightarrow \gamma^{*} / \mathrm{J} / \psi X \rightarrow \mu^{+} \mu^{-} X$ ), that is if the $q \bar{q}$ annihilation $\mathrm{J} / \psi$ production is dominating over $g g$ fusion $\mathrm{J} / \psi$ production in the COMPASS phase space, there is the possibility to study the polarised $\mathrm{J} / \psi$ cross-section and extract the TMD PDFs with much larger statistics. If on the other hand $g g$ production mechanism is dominating, in principle it is still possible to extract the gluon Sivers TMD.

Considering a $\pi^{-}$beam of $190 \mathrm{GeV} / c$ with intensity $\mathrm{I}_{\text {beam }}=6 \times 10^{7}$ particles/s and an instantaneous luminosity $\mathrm{L}=1.2 \times 10^{32} \mathrm{~cm}^{-2} \mathrm{~s}^{-1}$, a DY event rate of 4300 events/day is expected in the IMR, a DY $+\mathrm{J} / \psi$ event rate of 25900 events/day in $2.9<\mathrm{M}_{\mu \mu}<3.2 \mathrm{GeV} / c^{2}$, and a DY event rate of 900 events/day in the HMR. Thus for two years of data taking (about 280 days) $250 \mathrm{k}$ events in the HMR are expected and the statistical errors for azimuthal asymmetries presented in Table 1. After 2 years of data taking our statistical accuracy is enough to have the asymmetries in bins of $x_{F}\left(x_{p}\right)$ and $p_{T}$.

Different theory predictions for the spin asymmetries in COMPASS are available. Figure 3 shows two Sivers predictions, one by Anselmino et al at the time of the proposal [4], presented together with the COMPASS statistical accuracy reached in 2 years of data-taking, and divided in $5 x_{F}$ bins; and a 
Table 1. Statistical errors of the azimuthal asymmetries, assuming 280 days of data-taking.

\begin{tabular}{|c|c|c|c|}
\hline $\begin{array}{c}\text { Asymmetry } \\
\text { error }\end{array}$ & $\begin{array}{c}2<\mathrm{M}_{\mu \mu}<2.5 \\
\mathrm{GeV} / c^{2}\end{array}$ & $\begin{array}{c}2.9<\mathrm{M}_{\mu \mu}<3.2 \\
\mathrm{GeV} / c^{2}\end{array}$ & $\begin{array}{c}4<\mathrm{M} f_{\mu \mu}<9 \\
\mathrm{GeV} / c^{2}\end{array}$ \\
\hline$\delta \mathrm{A}_{U}^{\cos 2 \phi}$ & 0.0026 & 0.0011 & 0.0057 \\
\hline$\delta \mathrm{A}_{T}^{\sin \phi_{S}}$ & 0.0065 & 0.0027 & 0.0143 \\
\hline$\delta \mathrm{A}_{T}^{\sin \left(2 \phi+\phi_{S}\right)}$ & 0.0130 & 0.0053 & 0.0285 \\
\hline$\delta \mathrm{A}_{T}^{\sin \left(2 \phi-\phi_{S}\right)}$ & 0.0130 & 0.0053 & 0.0285 \\
\hline
\end{tabular}
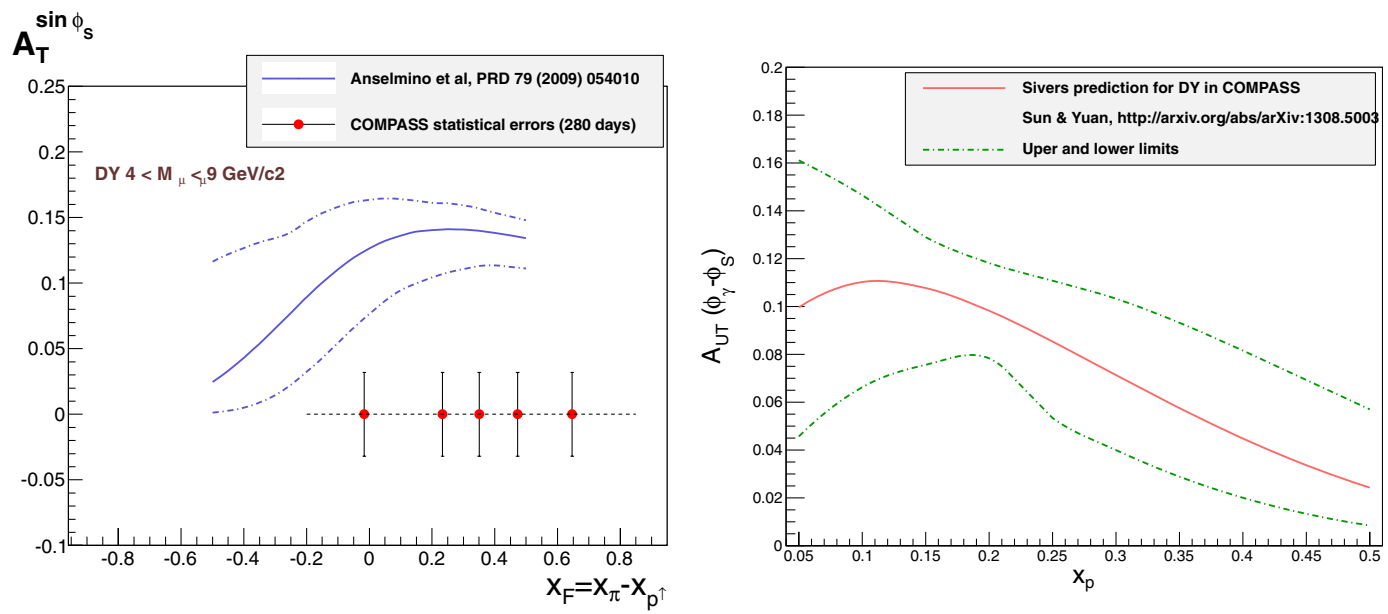

Figure 3. Two of the existing Sivers asymmetry predictions for the COMPASS case. (a) Sivers prediction from Anselmino et al from Proposal [4] and COMPASS statistical accuracy considering 2 years of data taking divided in $5 x_{F}$ bins. (b) Sivers prediction from Sun and Yuan in terms of $x_{\mathrm{p}}$ considering the COMPASS phase-space [6].

second prediction by Sun and Yuan recently provided [6]. Both are around 10\% in the region where our acceptance is larger. COMPASS has a geometrical acceptance in the HMR of $39 \%$.

\section{Summary}

The opportunity to study in the same experiment the TMD PDFs from both SIDIS and the DY processes is unique and will allow to check the predicted sign change in Sivers and Boer-Mulders functions when accessed by either of these processes after one year of data taking. After two years of data taking there will be enough statistics to study the asymmetries in terms of $x_{F}\left(x_{\mathrm{p}}\right)$ and $p_{T}$. The feasibility of the measurement has been proved by several tests already performed. The polarised Drell-Yan experiment will start in the end of 2014 and continue during 2015. A second year of DY data taking is planned, possibly in 2018. There are several worldwide plans to study TMD PDFs via the polarised DY process and COMPASS aims to perform the first polarised DY experiment in the world.

This work was partially supported by the Portuguese Fundação para a Ciância e a Tecnologia. 


\section{References}

[1] S. Falciano et al. [NA10 Coll.], Z. Phys. C 31, 513 (1986)

[2] J.S. Conway et al. [E615 Coll.], Phys. Rev. D 39, 92 (1989)

[3] P. Abbon et al. [COMPASS Coll.], Nucl. Inst. Meth. A 577, 455 (2007)

[4] COMPASS-II proposal, CERN-SPSC-2010-014, SPSCP-340, May 2010

[5] J.C. Collins, Phys. Lett. B 536, 43 (2002)

[6] http://arxiv.org/abs/arXiv:1308.5003 